\title{
The Mazurkiewicz distance and sets that are finitely connected at the boundary
}

\author{
Anders Björn \\ Department of Mathematics, Linköpings universitet, \\ SE-58183 Linköping, Sweden; anders.bjorn@liu.se \\ Jana Björn \\ Department of Mathematics, Linköpings universitet, \\ SE-58183 Linköping, Sweden; jana.bjorn@liu.se \\ Nageswari Shanmugalingam \\ Department of Mathematical Sciences, University of Cincinnati, \\ P.O. Box 210025, Cincinnati, OH 45221-0025, U.S.A.; shanmun@uc.edu
}

\begin{abstract}
We study local connectedness, local accessibility and finite connectedness at the boundary, in relation to the compactness of the Mazurkiewicz completion of a bounded domain in a metric space. For countably connected planar domains we obtain a complete characterization. It is also shown exactly which parts of this characterization fail in higher dimensions and in metric spaces.
\end{abstract}

Key words and phrases: compactness, countably connected planar domain, finitely connected at the boundary, locally accessible, locally connected, Mazurkiewicz boundary, metric space.

Mathematics Subject Classification (2010): 54D05 (Primary), 54E35, 30L99, 30D40 (Secondary).

\section{Introduction}

In this paper we study the question of compactness of the completion of a domain $\Omega$ with respect to the Mazurkiewicz distance

$$
d_{M}(x, y)=\inf \operatorname{diam} E,
$$

where the infimum is taken over all connected sets $E \subset \Omega$ containing $x, y \in \Omega$. The distance is also called relative distance, Mazurkiewicz intrinsic metric and inner diameter distance in the literature. We characterize the compactness of this completion using topological properties of the domain near the boundary, such as finite connectivity at the boundary, local accessibility and local connectivity of the boundary. Even in the Euclidean setting these topological notions are delicate, and some characterizations hold only in the two-dimensional setting.

This study is part of a project which deals with extending the notion of boundary in connection with the Dirichlet problem for partial differential operators and variational problems, see Adamowicz-Björn-Björn-Shanmugalingam [1] and BjörnBjörn-Shanmugalingam [6]. The Euclidean boundary is usually sufficient for smooth enough domains, but for more general domains a different notion of boundary is 
needed. For example, even in the simple case of a planar slit disc, points on the slit can be approached from two different directions, and one should permit the Dirichlet solution to take two different boundary values corresponding to these different approaches to that point. The Mazurkiewicz boundary considered in this paper partially rectifies this issue, but does not solve the problem completely. More concretely, in [6] the Perron method for solving the Dirichlet problem for $p$-harmonic functions was developed with respect to the Mazurkiewicz boundary, under the assumption that it is compact. (For the corresponding problem with respect to the Euclidean boundary (on $\mathbf{R}^{n}$ ) or given metric boundary (on metric spaces), see [10], [16], [11], [4], [5] and [3], as well as the references in the notes to [11, Sections 9 and 16].) Therefore the compactness of the Mazurkiewicz boundary is of particular interest and importance to us.

In the general setting of proper locally pathconnected metric spaces we prove the following result. See Section 2 for the definitions of the involved notions.

Theorem 1.1. The closure $\bar{\Omega}^{M}$ with respect to the Mazurkiewicz distance is compact if and only if $\Omega$ is finitely connected at the boundary.

Finite connectivity of $\Omega$ at a boundary point $x_{0}$ is also characterized in terms of components of $\Omega \cap B\left(x_{0}, r\right)$, see Proposition 2.5. Proposition 3.3 provides a connection between finite connectivity of $\Omega$ at $x_{0}$ and the number of points in the Mazurkiewicz boundary $\partial_{M} \Omega$ corresponding to $x_{0}$.

For countably connected planar domains we prove the following theorem, which generalizes a result by Newman [24], and follows from the more general Theorem 4.4.

Theorem 1.2. If $\Omega \subset \mathbf{R}^{2}$ and $\mathbf{R}^{2} \backslash \Omega$ has countably many components, then the following are equivalent:

(a) each $x_{0} \in \partial \Omega$ is locally accessible from $\Omega$;

(b) for every $x_{0} \in \partial \Omega$ we have that $\Omega \cup\left\{x_{0}\right\}$ is locally connected at $x_{0}$;

(c) for all $r>0$ and $x_{0} \in \partial \Omega$ it is true that $x_{0} \notin \overline{H\left(r, x_{0}\right)}$;

(d) $\Omega$ is finitely connected at the boundary;

(e) $\bar{\Omega}^{M}$ is compact.

For finitely connected planar domains we further show in Theorem 4.2 that (a)-(e) are equivalent to each of

(f) $\partial \Omega$ is locally connected and (g) $\mathbf{R}^{2} \backslash \Omega$ is locally connected,

while for infinitely connected planar domains both (f) and (g) always fail, see Theorem 4.4. For a general bounded domain in a proper locally connected metric space we only obtain the following implications:

$$
(\mathrm{e}) \Longleftrightarrow(\mathrm{d}) \Longrightarrow(\mathrm{c}) \Longleftrightarrow(\mathrm{b}) \Longleftrightarrow(\mathrm{a}) \text { and }(\mathrm{f}) \Longrightarrow(\mathrm{g})
$$

see Theorem 4.3, We also construct Examples 4.8-4.11 showing that no other implication holds true even when $\Omega \subset \mathbf{R}^{3}$ is required to be homeomorphic to a ball.

In Adamowicz-Björn-Björn-Shanmugalingam [1, Theorem 9.6] it was shown that the Mazurkiewicz boundary coincides with the collection of singleton prime ends of the domain. Our results therefore also have applications in connection with prime ends. In particular, in Section 7 of [1], singleton prime ends are related to (nonlocal) accessibility of boundary points, while in Section 10 of [1] compactness of the singleton prime end boundary is discussed. (Beware that the prime ends introduced in [1] do not always coincide with the classical Carathéodory prime ends for simply connected planar domains.)

As to be expected, the discussion in this paper deals with purely metric topological notions, and hence might be of interest to a wider audience. Some parts of this 
paper are related to the results of Rempe [28], which characterizes local connectivity at the boundary in terms of prime ends for simply connected planar domains, and to R. L. Moore [20]. However, the reader should beware that the definitions of compactness, connectedness and continuum in [20] are different from the modern definitions.

The paper is organized as follows. In Section 2 we describe the topological notions used throughout the paper, while in Section 3 we define and discuss the Mazurkiewicz distance, and in particular prove Theorem 1.1. In Section 4 we describe relations between the finite connectivity at the boundary and other topological notions.

In the last section we revisit planar domains and show that a countably connected planar domain is finitely connected at the boundary whenever each topological boundary point is locally accessible from it (Theorem 5.7), generalizing a result by Newman [24].

Acknowledgement. This research was begun while the first two authors visited the University of Cincinnati during the first half year of 2010, and continued while the third author visited Linköpings universitet in March 2011, and during the stay of the three authors at Institut Mittag-Leffler in Autumn 2013. We wish to thank these institutions for their kind hospitality. We also wish to thank Tomasz Adamowicz and Harold Bell for fruitful discussions.

The first two authors were supported by the Swedish Research Council. The first author was also a Fulbright scholar during his visit to the University of Cincinnati, supported by the Swedish Fulbright Commission, while the second author was a Visiting Taft Fellow during her visit to the University of Cincinnati, supported by the Charles Phelps Taft Research Center at the University of Cincinnati. The third author was also supported by the Taft Research Center of the University of Cincinnati and by grant \#200474 from the Simons Foundation and NSF grant DMS-1200915.

\section{Local connectedness}

Throughout the paper we let $(X, d)$ be a metric space and let $\Omega \subset X$ be a bounded domain, i.e. $\Omega$ is a bounded nonempty open connected set.

In this section we describe various notions of connectedness which are useful in the study of topological properties of the Mazurkiewicz boundary. We also characterize finite and bounded connectivity of $\Omega$ at a boundary point $x_{0}$ in terms of components of $B\left(x_{0}, r\right) \cap \Omega$.

There are four distinct types of local connectedness one can consider at a point in a metric space.

Definition 2.1. A topological space $Y$ is locally (path) connected at $x \in Y$ if for every $r>0$ there is a (path)connected neighbourhood $G \subset B(x, r)$ of $x$.

The space $Y$ is (path)connected im kleinen at $x \in Y$ if for every $r>0$ there is a (path)connected set $A \subset B(x, r)$ such that $x \in \operatorname{int} A$.

We also say that $Y$ has any of these properties globally if it holds for all $x \in Y$.

Here $B(x, r)=\{y \in X: d(x, y)<r\}$ and neighbourhoods are open.

The terminology is not standardized throughout the literature. Indeed, Kuratowski's definition in [17] of local (arcwise) connectedness corresponds to the above definition of (path)connectedness im kleinen. Whyburn's definition in [30] of local connectedness is our definition of connectedness im kleinen. We follow the terminology of Munkres [21] in defining local connectedness. However, [21] uses the name weakly locally connected instead of connected im kleinen. 
It is obvious that we have the following implications:

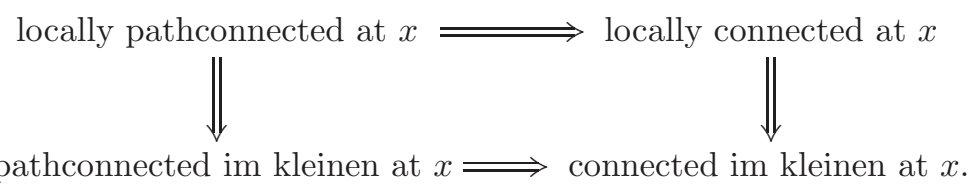

pathconnected im kleinen at $x \Longrightarrow$ connected im kleinen at $x$.

For the corresponding global statements (i.e. the assumptions are required at all $x \in X)$ it is known that also the corresponding upward implications hold. Indeed, if $X$ is (path)connected im kleinen at every $x \in X$ and $r>0$, then the (path)connected component of $B(x, r)$ containing $x$ must be open, see e.g. Whyburn [30, p. 20, (14.1)], and hence $X$ is locally (path)connected at $x$.

If $X$ is moreover complete, then all four global statements are equivalent by Mazurkiewicz-Moore-Menger's theorem, see Kuratowski [17, p. 154, Theorem 1]. On the other hand, Moore [19] gave an example of a locally connected metric space which is not locally pathconnected. In fact his example is a noncomplete subset of $\mathbf{R}^{2}$ which is connected and locally connected but contains no path.

We are mostly interested in considering connectedness properties at points on the boundary of $\Omega$. Fix a boundary point $x_{0} \in \partial \Omega$ throughout the rest of this section.

Definition 2.2. We say that $\Omega$ is finitely connected at $x_{0} \in \partial \Omega$ if for every $r>0$ there is an open set $G \subset X$ such that $x_{0} \in G \subset B\left(x_{0}, r\right)$ and $G \cap \Omega$ has only finitely many components.

If there is $N>0$ such that for every $r>0$ there is an open $G \subset X$ such that $x_{0} \in G \subset B\left(x_{0}, r\right)$ and such that $G \cap \Omega$ has at most $N$ components, then we say that $\Omega$ is boundedly connected at $x_{0}$. If moreover $N$ is minimal, then $\Omega$ is said to be $N$-connected at $x_{0}$. Furthermore, $\Omega$ is locally connected at $x_{0} \in \partial \Omega$ if it is 1-connected at $x_{0}$.

We say that $\Omega$ has one of these properties at the boundary if it has that property at all boundary points.

The terminology above follows Näkki [22] who seems to have first used this terminology in print. (Näkki [23] has informed us that he learned about the terminology from Väisälä, who however first seems to have used it in print in [29].) The concept of finite connectedness at the boundary was already used by Newman [24] (only in the first edition), but without a name.

Beware that the notion of finitely connected domains is a completely different notion (also used later in this paper). Similarly, it is important to distinquish between "locally connected", "locally connected at a point" (as in Definition 2.1), "locally connected at a boundary point" and "locally connected at the boundary" (as in Definition 2.2). For example, note that for $\Omega$ to be locally connected at $x_{0} \in \partial \Omega$ (in the sense of Definition 2.2) it is necessary that $\Omega \cup\left\{x_{0}\right\}$ is locally connected at $x_{0}$ (in the sense of Definition 2.1). The converse need not hold, as evidenced by the slit $\operatorname{disc} \Omega=\left\{(x, y) \in \mathbf{R}^{2}: x^{2}+y^{2}<1\right\} \backslash([0,1) \times\{0\})$, with $x_{0}=\left(\frac{1}{2}, 0\right) \in \mathbf{R}^{2}$. See Theorem 4.12 for the relations between these various notions of connectedness.

Theorems 1.10 and 1.11 of [22] give several characterizations of finite connectedness and $N$-connectedness. We provide some further characterizations in this section, which will be useful for us in the study of the Mazurkiewicz boundary. The results of [22] are formulated for $\overline{\mathbf{R}^{n}}$, but the proofs of these topological properties given there also hold in metric spaces.

Example 2.3. The following was shown in Adamowicz-Björn-Björn-Shanmugalingam [1]. (See [1] for definitions of the concepts mentioned above and below.) The following are true: 
(a) If $\Omega$ is a uniform domain, then $\Omega$ is locally connected at the boundary.

(b) If $\Omega$ is finitely connected at the boundary and $X$ is locally connected and proper, then there is a natural one-to-one correspondence between the points in the Mazurkiewicz boundary (see Definition 3.2) and the prime end boundary using the new definition of prime ends introduced in [1].

If $X$ is a quasiconvex proper metric space equipped with a doubling measure $\mu$, then the following are true:

(c) If $\Omega$ is a John domain, then $\Omega$ is boundedly connected at the boundary.

(d) If $\Omega$ is an almost John domain, then $\Omega$ is finitely connected at the boundary.

For each $r>0$ let $\left\{G_{j}\left(r, x_{0}\right)\right\}_{j=1}^{N\left(r, x_{0}\right)}$ be the components of $B\left(x_{0}, r\right) \cap \Omega$ that have $x_{0}$ in their boundary, i.e. $x_{0} \in \overline{G_{j}\left(r, x_{0}\right)}$. Here $N\left(r, x_{0}\right)$ is either a nonnegative integer or $\infty$, with $N\left(r, x_{0}\right)=0$ indicating that there is no connected component of $B\left(x_{0}, r\right) \cap \Omega$ that has $x_{0}$ in its boundary. Further, let

$$
H\left(r, x_{0}\right)=B\left(x_{0}, r\right) \cap \Omega \backslash \bigcup_{j=1}^{N\left(r, x_{0}\right)} G_{j}\left(r, x_{0}\right)
$$

be the union of the remaining components (if any). We will often drop $x_{0}$ from the notation when it is clear from the context which point is under consideration.

Lemma 2.4. If $x_{0} \notin \overline{H(r)}$ for every $r>0$, then $N(\cdot)$ is a nonincreasing function.

Observe that if $0<r<s$ and $x_{0} \notin \overline{H(r)}$, then $x_{0} \notin \overline{H(s)}$.

Proof. Let $0<r<s$. As $x_{0} \notin H(s)$ there is at least one component $G_{1}(s)$. Fix any component $G_{j}(s)$. As $x_{0} \in \overline{G_{j}(s)}$, we have that $G_{j}(s) \cap B\left(x_{0}, r\right)$ is nonempty and thus consists of one or more components. Not all of these components can lie in $H(r)$ as $x_{0} \notin \overline{H(r)}$. Hence there is $k(j)$ such that $G_{k(j)}(r) \subset G_{j}(s) \cap B\left(x_{0}, r\right)$. As the $G_{j}(s)$ are pairwise disjoint, $k(\cdot)$ must be injective, and thus $N(s) \leq N(r)$.

Proposition 2.5. The set $\Omega$ is finitely connected at $x_{0}$ if and only if for all $r>0$, $N(r)<\infty$ and $x_{0} \notin \overline{H(r)}$.

As the following example illustrates, having $0<N(r)<\infty$ for all $r>0$ by itself does not guarantee finite connectivity.

Example 2.6. (The topologist's comb I) Let $\Omega \subset \mathbf{R}^{2}$ be given by

$$
\Omega:=((-1,1) \times(0,2)) \backslash\left(\left\{\frac{1}{2}, \frac{1}{3}, \frac{1}{4}, \ldots, 0\right\} \times(0,1]\right)
$$

and $x_{0}=(0,0)$. Then $N(r)=1$ for all $r>0$, but $\Omega$ is not finitely connected at $x_{0}$. Note that $\Omega$ is simply connected.

Proof of Proposition 2.5. Assume first that $\Omega$ is finitely connected at $x_{0}$, and let $r>0$. Then there is a neighbourhood $G \subset B\left(x_{0}, r\right)$ of $x_{0}$ such that $G \cap \Omega$ has only finitely many components. It follows that $x_{0} \notin \overline{H(r)}$, as otherwise $G \cap H(r)$ would have infinitely many components. Furthermore, as $x_{0} \in \overline{G_{j}(r)}$ we must have that $G \cap G_{j}(r) \neq \varnothing$ for every $j$. Moreover, two different components $G_{j}(r)$ and $G_{k}(r)$ cannot intersect the same component of $G \cap \Omega$, as $G \subset B\left(x_{0}, r\right)$. Thus $N(r)$ is no larger than the number of components of $G$, which is finite by assumption.

Conversely, let $r>0$ and assume that $N(r)<\infty$ and $x_{0} \notin \overline{H(r)}$. Then there is $0<s<r$ such that $B\left(x_{0}, s\right) \cap H(r)=\varnothing$. It follows that $G=B\left(x_{0}, s\right) \cup$ $\bigcup_{j=1}^{N(r)} G_{j}(r) \subset B\left(x_{0}, r\right)$ is a neighbourhood of $x_{0}$ such that $G \cap \Omega=\bigcup_{j=1}^{N(r)} G_{j}(r)$ has only $N(r)$ number of components. 
Proposition 2.7. The set $\Omega$ is $N$-connected at $x_{0}$ if and only if $x_{0} \notin \overline{H(r)}$ for all $r>0$ and $\lim _{r \rightarrow 0} N(r)=N$. Moreover, in this case there is $r_{0}>0$ such that $N\left(r_{0}\right)=N$.

Proof. The proof is similar to the proof of Proposition 2.5 with some extra details. Assume first that $\Omega$ is $N$-connected at $x_{0}$. Just as in the proof of Proposition 2.5 we find that for all $r>0$ it is true that $x_{0} \notin \overline{H(r)}$ and that $N(r) \leq N$. As $N(\cdot)$ is nonincreasing the latter condition is equivalent to the condition $\lim _{r \rightarrow 0} N(r) \leq N$. Since $N$ is the minimal such integer, it follows that $\lim _{r \rightarrow 0} N(r)=N$.

Conversely, by Lemma 2.4, $N(r) \leq \lim _{r \rightarrow 0} N(r)$ for all $r>0$. Thus, the proof of Proposition 2.5 shows that in this case $\Omega$ is at most $\lim _{r \rightarrow 0} N(r)$-connected at $x_{0}$. It follows that we also must have $N=\lim _{r \rightarrow 0} N(r)$. As $N(\cdot)$ is decreasing and integer-valued there must be some $r_{0}>0$ such that $N\left(r_{0}\right)=N$.

Corollary 2.8. The domain $\Omega$ is boundedly connected at $x_{0}$ if and only if $x_{0} \notin \overline{H(r)}$ for all $r>0$ and $\lim _{r \rightarrow 0} N(r)<\infty$.

This corollary follows directly from Proposition 2.7.

\section{The Mazurkiewicz distance $d_{M}$}

From now on, we assume in this paper that $X$ is a proper (i.e. closed bounded sets are compact) locally connected metric space.

Since $X$ is proper it is complete, and thus Mazurkiewicz-Moore-Menger's theorem shows that $X$ is locally pathconnected, see Section 2 .

Definition 3.1. We define the Mazurkiewicz distance $d_{M}$ on $\Omega$ by

$$
d_{M}(x, y)=\inf \operatorname{diam} E,
$$

where the infimum is taken over all connected sets $E \subset \Omega$ containing $x, y \in \Omega$.

For comparison, let us further define the inner metric $d_{\text {inner }}$ on $\Omega$ by

$$
d_{\text {inner }}(x, y)=\inf l_{\gamma}
$$

where the infimum is taken over all rectifiable curves $\gamma:\left[0, l_{\gamma}\right] \rightarrow \Omega$ parameterized by arc length such that $\gamma(0)=x$ and $\gamma\left(l_{\gamma}\right)=y$.

The Mazurkiewicz distance $d_{M}$ is always a metric on $\Omega$ and, because $X$ is locally connected, it generates the same topology as $d$ on $\Omega$. Furthermore, we have $d \leq$ $d_{M} \leq d_{\text {inner }}$. The function $d_{\text {inner }}$ is a metric if and only if $\Omega$ is rectifiably connected, i.e. any two points are connected by a rectifiable curve (a curve with finite length) in $\Omega$.

The Mazurkiewicz distance was introduced by Mazurkiewicz [18], in relation to a classification of points on $n$-dimensional Euclidean continua. The metric $d_{M}$ goes under different names in the literature, it is e.g. denoted $\rho_{A}$ in [18], called relative distance and denoted $\varrho_{r}$ in Kuratowski [17], called Mazurkiewicz intrinsic metric and denoted $\delta_{D}$ in Karmazin [15], denoted $\mu$ in Newman [24] (only in the first edition), and by $m$ in Ohtsuka [25]. In Aikawa-Hirata [2], Freeman-Herron [9] and Herron-Sullivan [12] it is called inner diameter distance. Here and in AdamowiczBjörn-Björn-Shanmugalingam [1] and Björn-Björn-Shanmugalingam [6] we call it the Mazurkiewicz distance.

Definition 3.2. The completion of the metric space $\left(\Omega, d_{M}\right)$ is denoted $\bar{\Omega}^{M}$. Furthermore, the Mazurkiewicz boundary is $\partial_{M} \Omega:=\bar{\Omega}^{M} \backslash \Omega$. 
Note that each point $y \in \partial_{M} \Omega$ corresponds to (an equivalence class of) a sequence $\left\{x_{j}\right\}_{j=1}^{\infty}$ which is a Cauchy sequence with respect to the Mazurkiewicz metric $d_{M}$ and which does not have a limit point in $\Omega$.

As advertised in Theorem 1.1, the closure $\bar{\Omega}^{M}$ is compact if and only if $\Omega$ is finitely connected at the boundary. This result was proven by Karmazin $[15$, Theorem 1.3.8], but the proof is available only in Russian and not widely accessible. For the reader's convenience, we give a more elementary self-contained proof which appeals only to basic definitions.

Proof of Theorem 1.1. First assume that $\Omega$ is finitely connected at the boundary. As $\bar{\Omega}^{M}$ is a metric space, it is compact if and only if it is sequentially compact, see e.g. Munkres [21, Theorem 28.2].

Let $\left\{y_{j}\right\}_{j=1}^{\infty}$ be a sequence in $\bar{\Omega}^{M}$. For each $j$ we can find $x_{j} \in \Omega$ such that $d_{M}\left(x_{j}, y_{j}\right)<1 / j$. Then $\left\{x_{j}\right\}_{j=1}^{\infty}$ has a convergent subsequence if and only if $\left\{y_{j}\right\}_{j=1}^{\infty}$ has, and it is thus enough to show that every sequence $\left\{x_{j}\right\}_{j=1}^{\infty}$ from $\Omega$ has a convergent subsequence in $\bar{\Omega}^{M}$. To this end, let $\left\{x_{j}\right\}_{j=1}^{\infty}$ be a sequence in $\Omega$. Because $X$ is proper and $\Omega$ is bounded, this sequence has a cluster point $x_{0} \in \bar{\Omega}$ with respect to the original metric $d$. By taking a subsequence if necessary we may assume that $x_{j} \stackrel{d}{\rightarrow} x_{0}$ as $j \rightarrow \infty$. If $x_{0} \in \Omega$, then the local connectivity of $X$ implies that $x_{j} \stackrel{d_{M}}{\longrightarrow} x_{0}$ as $j \rightarrow \infty$, and we are done. Assume therefore that $x_{0} \in \partial \Omega$.

By assumption, $\Omega$ is finitely connected at $x_{0}$, and in particular $N(r)<\infty$ for all $r>0$. There must therefore be some $k_{0}$ such that infinitely many of the $x_{j}$ belong to $G_{k_{0}}(1)$. Collect them in a subsequence $\left\{x_{j}^{(0)}\right\}_{j=1}^{\infty}$. Similarly, there is some $k_{1}$ such that infinitely many of the $x_{j}^{(0)}$ belong to $G_{k_{1}}\left(\frac{1}{2}\right)$. Again, collect them in a subsequence $\left\{x_{j}^{(1)}\right\}_{j=1}^{\infty}$. Proceeding inductively, we find $k_{2}, k_{3}, \ldots$, and further and further refined subsequences $\left\{x_{j}^{(2)}\right\}_{j=1}^{\infty},\left\{x_{j}^{(3)}\right\}_{j=1}^{\infty}, \ldots$ such that $x_{j}^{(m)} \in G_{k_{m}}\left(2^{-m}\right)$. A diagonalization argument lets us choose $z_{j}=x_{j}^{(j)}$, a subsequence of the original sequence. Now, if $m>j$, then

$$
d_{M}\left(z_{j}, z_{m}\right) \leq \operatorname{diam} G_{k_{j}}\left(2^{-j}\right) \leq \operatorname{diam} B\left(x_{0}, 2^{-j}\right) \leq 2^{1-j} .
$$

Hence $\left\{z_{j}\right\}_{j=1}^{\infty}$ is a Cauchy sequence in the metric $d_{M}$ and thus has a limit in $\bar{\Omega}^{M}$.

Conversely, assume that $\Omega$ is not finitely connected at the boundary. Then there is some $x_{0} \in \partial \Omega$ such that $\Omega$ is not finitely connected at $x_{0}$. By Proposition 2.5, there is some $r>0$ such that either $N(r)=\infty$ or $x_{0} \in \overline{H(r)}$.

If $N(r)=\infty$, then we can find $x_{j} \in G_{j}(r) \cap B\left(x_{0}, \frac{1}{2} r\right), j=1,2, \ldots$. If $j \neq k$ and $x_{j}, x_{k} \in E \subset \Omega$ for some connected set $E$, then by construction $E$ must contain a point outside $B\left(x_{0}, r\right)$, and hence $\operatorname{diam} E \geq r / 2$ showing that $d_{M}\left(x_{j}, x_{k}\right) \geq r / 2$. This implies that $\left\{x_{j}\right\}_{j=1}^{\infty}$ cannot have a convergent subsequence with respect to $d_{M}$.

Finally, if $x_{0} \in \overline{H(r)}$, then we can find points $x_{j} \in H(r) \cap B\left(x_{0}, r / 2\right)$ such that $x_{j}$ belongs to distinct components of $H(r)$ (and thus of $\Omega \cap B\left(x_{0}, r\right)$ ). The rest of the argument is identical to the reasoning of the case $N(r)=\infty$ above.

The following result and its proof give a good understanding of how the boundary $\partial_{M} \Omega$ looks like.

Proposition 3.3. Assume that $\Omega$ is $N$-connected at $x_{0}$. Then there are exactly $N$ boundary points in $\partial_{M} \Omega$ corresponding to $x_{0}$.

By saying that the point $y=\left\{x_{j}\right\}_{j=1}^{\infty} \in \partial_{M} \Omega$ corresponds to $x_{0}$, we mean that $x_{j} \stackrel{d}{\rightarrow} x_{0} \in \partial \Omega$. 
Proof. Without loss of generality we may assume that $N(1)=N$. By Lemma 2.4, $N(\cdot)$ is nonincreasing, and so $N(r)=N$ for $0<r \leq 1$. For $0<r<1$ each $G_{j}(r)$ belongs to one of the components $G_{k}(1)$, and we may assume that they have been ordered so that $G_{j}(r) \subset G_{j}(s)$ if $0<r<s \leq 1$. For each positive integer $j \leq N$, choose a sequence $x_{k} \in G_{j}(1)$ such that $x_{k} \stackrel{d}{\rightarrow} x_{0}$. As $x_{0} \in \overline{G_{j}(1)}$ there are such sequences. We shall show that $\left\{x_{k}\right\}_{k=1}^{\infty}$ has a limit in $\bar{\Omega}^{M}$.

Let $\varepsilon>0$. Then by Proposition 2.7, $x_{0} \notin \overline{H(\varepsilon)}$. Therefore there is $0<\delta<\varepsilon$ such that $B\left(x_{0}, \delta\right) \cap \overline{H(\varepsilon)}=\varnothing$. Moreover there is $K>1$ such that $d\left(x_{k}, x_{0}\right)<\delta$ for $k>K$. Thus, for $k>K, x_{k} \in G_{j(k)}(\varepsilon)$ for some $j(k)$, and as $x_{k} \in G_{j}(1)$ we must have $j(k)=j$. Hence for $k, l>K$,

$$
d_{M}\left(x_{k}, x_{l}\right) \leq \operatorname{diam} G_{j}(\varepsilon) \leq 2 \varepsilon .
$$

Thus $\left\{x_{k}\right\}_{k=1}^{\infty}$ is a Cauchy sequence in the $d_{M}$-metric and has a limit in $\bar{\Omega}^{M}$.

As $\left\{x_{k}\right\}_{k=1}^{\infty}$ was an arbitrary sequence in $G_{j}(1)$ with $x_{k} \stackrel{d}{\rightarrow} x_{0}$, all such sequences must have the same limit in $\bar{\Omega}^{M}$, denoted $y_{j}$.

Now take $\partial_{M} \Omega \ni y=\left\{x_{k}\right\}_{k=1}^{\infty}$, where $\left\{x_{k}\right\}_{k=1}^{\infty}$ is a sequence in $\Omega$ that is Cauchy with respect to the metric $d_{M}$ and in addition $x_{k} \stackrel{d}{\rightarrow} x_{0}$. As $N$ is finite and $x_{0} \notin \overline{H(1)}$ there is an integer $j$ and a subsequence $\left\{x_{k_{l}}\right\}_{l=1}^{\infty}$ such that $x_{k_{l}} \in G_{j}(1)$ for all $l$. It follows that $x_{k_{l}} \stackrel{d_{M}}{\longrightarrow} y_{j}$. Hence $y=y_{j}$ and $x_{k} \stackrel{d_{M}}{\longrightarrow} y_{j}$.

Thus $y_{1}, \ldots, y_{N}$ are all the boundary points in $\bar{\Omega}^{M}$ corresponding to $x_{0}$. It remains to show that they are all distinct. Let $1 \leq j<k \leq N$. By the construction of $y_{j}$ we can find $x_{j} \in G_{j}(1)$ such that $d_{M}\left(x_{j}, y_{j}\right)<\frac{1}{4}$, and similarly $x_{k} \in G_{k}(1)$ such that $d_{M}\left(x_{k}, y_{k}\right)<\frac{1}{4}$. In particular $d\left(x_{j}, y_{j}\right)<\frac{1}{4}$ and $d\left(x_{k}, y_{k}\right)<\frac{1}{4}$. Let $F \subset \Omega$ be any connected open set containing $x_{j}$ and $x_{k}$. As $x_{j}$ and $x_{k}$ belong to different components of $B\left(x_{0}, 1\right) \cap \Omega$, the set $F$ must contain a point $z \in \Omega \backslash B\left(x_{0}, 1\right)$. Hence

$$
\operatorname{diam} F \geq d\left(z, x_{j}\right) \geq d\left(z, x_{0}\right)-d\left(x_{0}, x_{j}\right)>1-\frac{1}{4}=\frac{3}{4},
$$

and thus $d_{M}\left(x_{j}, x_{k}\right) \geq \frac{3}{4}$. So

$$
d_{M}\left(y_{j}, y_{k}\right) \geq d_{M}\left(x_{j}, x_{k}\right)-d_{M}\left(x_{j}, y_{j}\right)-d_{M}\left(x_{k}, y_{k}\right)>\frac{3}{4}-\frac{1}{4}-\frac{1}{4}=\frac{1}{4}
$$

If $\Omega$ is finitely but not boundedly connected at $x_{0}$ one similarly gets infinitely many points in $\partial_{M} \Omega$ corresponding to $x_{0}$. The proof of this fact is similar to the proof above.

We define $\Phi: \bar{\Omega}^{M} \rightarrow \bar{\Omega}$ in the following way: For $x \in \Omega$ we let $\Phi(x)=x$. Since $d \leq d_{M}$, this is a 1-Lipschitz map on $\Omega$. Because $\bar{\Omega}$ is complete, this map has a unique continuous extension to $\bar{\Omega}^{M}$, which we again denote by $\Phi$, which is also 1-Lipschitz. If $x_{0} \in \partial \Omega$ and $\Omega$ is $N$-connected at $x_{0}$, then $\Phi^{-1}\left(x_{0}\right)$ consists of the $N$ points called $y_{1}, \ldots, y_{N}$ in the proof above.

\section{Various types of local connectedness at the bound- ary}

For a boundary point $x_{0} \in \partial \Omega$ there are four types of local connectedness one can discuss: that $\Omega$ is locally connected at $x_{0}$, that $\Omega \cup\left\{x_{0}\right\}$ is locally connected at $x_{0}$, that $\partial \Omega$ is locally connected at $x_{0}$ and that $X \backslash \Omega$ is locally connected at $x_{0}$. The last three conditions come in four variants according to Definition 2.1, while for the first we have just one variant (given in Definition 2.2). These properties are closely related and also closely related to the compactness of $\bar{\Omega}^{M}$. 
Let us start with the following characterization valid for finitely connected planar domains. We first recall the definition of local accessibility.

Definition 4.1. A boundary point $x_{0} \in \partial \Omega$ is locally accessible from $\Omega$ if for every $r>0$ there is $\delta>0$ such that for every $x \in B\left(x_{0}, \delta\right) \cap \Omega$ there is a (not necessarily rectifiable) curve $\gamma:[0,1] \rightarrow X$ such that $\gamma([0,1)) \subset B\left(x_{0}, r\right) \cap \Omega, \gamma(0)=x$ and $\gamma(1)=x_{0}$.

See also the related definitions about connectivity given in Definition 2.1.

The following theorem (except for $(\mathrm{g})$ ) was proven for simply connected planar domains by Newman in the first edition of the book [24] (but was omitted from the second edition of [24]).

Theorem 4.2. Assume that $\Omega \subset \mathbf{R}^{2}$ is a bounded and finitely connected domain. Then the following are equivalent:

(a) each $x_{0} \in \partial \Omega$ is locally accessible from $\Omega$;

(b) for every $x_{0} \in \partial \Omega$ we have that $\Omega \cup\left\{x_{0}\right\}$ is locally connected at $x_{0}$;

(c) for all $r>0$ and $x_{0} \in \partial \Omega$ it is true that $x_{0} \notin \overline{H\left(r, x_{0}\right)}$;

(d) $\Omega$ is finitely connected at the boundary;

(e) $\bar{\Omega}^{M}$ is compact;

(f) $\partial \Omega$ is locally connected;

(g) $\mathbf{R}^{2} \backslash \Omega$ is locally connected.

Since $\Omega \subset \mathbf{R}^{2}$, it is finitely (simply) connected if and only if $\mathbf{S}^{2} \backslash \Omega$ has only finitely many (one) components, where $\mathbf{S}^{2}$ is the Riemann sphere. Similar characterizations hold for the notions of countable, uncountable, and infinite connectedness of plane domains.

Observe that we can equivalently rephrase (b), (f) and (g) using any of the other three types of local connectedness in Definition 2.1. For (f) and (g) the equivalence of the four notions follows from the Mazurkiewicz-Moore-Menger theorem, see Section 2. The Mazurkiewicz-Moore-Menger theorem is however not available for (b) since $\Omega \cup\left\{x_{0}\right\}$ is not complete, so in this case we instead have to appeal to Theorem 4.12 below.

In view of $(\mathrm{d})$ and $(\mathrm{f})$ it is also natural to ask if the above statements could be equivalent to the statement " $\bar{\Omega}$ is locally connected" as well. That $\bar{\Omega}$ is locally connected follows from the other statements, which can be shown in the same way as the implication ( $\left.\mathrm{f} 2^{\prime}\right) \Rightarrow\left(\mathrm{g} 2^{\prime}\right)$ in Theorem 4.12 . However, it does not imply the other statements, as shown by the topologist's comb in Example 2.6. We therefore leave it out of the rest of the discussion.

Before proving Theorem 4.2, let us consider how much of this result is valid for more general domains $\Omega$ (i.e. infinitely connected in $\mathbf{R}^{2}$ as well as general domains in $\mathbf{R}^{n}$ and metric spaces).

Theorem 4.3. Consider the same statements as in Theorem 4.2 for a general bounded domain $\Omega$ in a proper locally connected metric space $X$ (with $\mathbf{R}^{2}$ replaced by $X$ in $(\mathrm{g}))$.

Then the following implications are true:

$$
\text { (e) } \Longleftrightarrow(\mathrm{d}) \Longrightarrow(\mathrm{c}) \Longleftrightarrow(\mathrm{b}) \Longleftrightarrow \text { (a) and }(\mathrm{f}) \Longrightarrow(\mathrm{g}) \text {. }
$$

No other implication is true even if we assume that $\Omega \subset \mathbf{R}^{3}$ is homeomorphic to a ball.

In $\mathbf{R}^{2}$ a little more is true, even if $\Omega$ is not simply connected. 
Theorem 4.4. Consider the same statements as in Theorem 4.2 for an infinitely connected bounded domain $\Omega \subset \mathbf{R}^{2}$. Then (f) and (g) fail.

If $\Omega$ is moreover countably connected, i.e. $\mathbf{R}^{2} \backslash \Omega$ has only countably many components, then (a)-(e) are equivalent. This holds also if $\mathbf{R}^{2} \backslash \Omega$ consists of at most countably many continua $K_{j}, j=1,2, \ldots$, and (possibly uncountably many) singleton components, and in addition $\bar{E} \backslash E$ is a union of a compact set and an at most countable set, where $E=\bigcup_{j=1}^{\infty} K_{j}$.

Recall that a continuum $K \subset \mathbf{R}^{2}$ is an infinite connected compact set. We postpone the proof of Theorem 4.4 until after the proofs of Theorems 4.2 and 4.3 later in this section.

We do not know whether (a) $\Rightarrow$ (e) always hold if $\Omega$ is an uncountably connected planar domain. The following example shows that it is possible for the statements (a)-(e) to be true even if $\Omega$ is infinitely connected.

Example 4.5. Let $C \subset[0,1]$ be the ternary Cantor set and $C_{j}$ be the $j$ th generation generating $C$ (i.e. $C=\bigcap_{j=0}^{\infty} C_{j}$ and $C_{j}$ consists of $2^{j}$ intervals each of length $3^{-j}$ ). Let further

$$
\begin{aligned}
& K=(C \times\{0\}) \cup \bigcup_{j=0}^{\infty}\left(C_{j} \times\left\{2^{-j}\right\}\right), \\
& \Omega=(-2,2)^{2} \backslash K \text { and } \Omega^{\prime}=\Omega \backslash([-1,0] \times\{0\}) .
\end{aligned}
$$

Then $\Omega$ and $\Omega^{\prime}$ are both uncountably connected domains and locally connected or 2connected at all their boundary points. Thus they both satisfy (a)-(e). Theorem 4.4 is however only applicable to $\Omega$, but not to $\Omega^{\prime}$ since in that case $\bar{E} \backslash E=(C \backslash\{0\}) \times\{0\}$ is not a union of a compact set and a countable set.

By replacing the line segments by thin rectangles it is easy to modify $\Omega$ and $\Omega^{\prime}$ so that they become locally connected at the boundary.

Open problem 4.6. Is Theorem 4.4 also true when $\bar{E} \backslash E$ is not a union of a compact set and an at most countable set?

If there are uncountably many continuum components of $\mathbf{R}^{2} \backslash \Omega$, then uncountably many of them have diameter larger than $\delta$, for some $\delta>0$, in which case it seems difficult to fulfill (a). This can be formulated as the following question.

Open problem 4.7. Does (a) always fail if there are uncountably many continuum components of $\mathbf{R}^{2} \backslash \Omega$ ? In view of Theorem 4.3 it would follow that (a)-(e) all fail, and are thus equivalent.

We now turn to the proof of Theorem 4.3.

Proof of Theorem 4.3. The implications (d) $\Rightarrow$ (c) $\Leftrightarrow(\mathrm{b}) \Leftrightarrow$ (a) and (f) $\Rightarrow$ (g) follow directly from the corresponding pointwise results in Theorem 4.12 below. The equivalence of $(\mathrm{d})$ and (e) is the content of Theorem 1.1.

Counterexamples for the failing implications are given as examples below, but we list them here. Note that they are all given for $\Omega \subset \mathbf{R}^{3}$ which is homeomorphic to a ball.

$(\mathrm{d}) \nRightarrow(\mathrm{g})$ This follows from Example 4.8.

(g) $\nRightarrow$ (f) This follows from Example 4.9 .

(f) $\nRightarrow$ (a) This follows from Example 4.10.

(a) $\nRightarrow$ (d) This follows from Example 4.11.

The failure of the remaining implications follows from these counterexamples in combination with the implications obtained in the beginning of the proof. 
The implication $(\mathrm{d}) \Rightarrow(\mathrm{g})$ is in general false. The following example shows that if $\Omega \subset \mathbf{R}^{3}$, then it is not enough to assume that $\Omega$ is homeomorphic to a ball. Note that $\Omega$ below is locally connected at the boundary.

Example 4.8. Let $G \subset \mathbf{R}^{2}$ be given by

$$
G:=((0,1) \times(-1,1)) \backslash \bigcup_{j=1}^{\infty} \overline{B\left(\left(2^{-j}, 0\right), 2^{-j} / 10\right)} .
$$

Further, let

$$
\Omega=\left((0,1) \times(-1,1)^{2}\right) \backslash(G \times[0,1)) .
$$

Then $\Omega$ is locally connected at the boundary, while $X \backslash \Omega$ is not locally connected at $(0,0,0)$. Note that $\Omega$ is homeomorphic to a ball.

In $\mathbf{R}^{3}$ we have the following example showing that $(\mathrm{g}) \not \Rightarrow(\mathrm{f})$. (It also shows that $(\mathrm{g}) \nRightarrow(\mathrm{a})$.)

Example 4.9. Let

$$
\Omega=(0,1)^{3} \cup \bigcup_{j=1}^{\infty}\left(\left(2^{-2 j-1}, 2^{-2 j}\right)^{2} \times(0,2)\right) .
$$

In this case $\mathbf{R}^{3} \backslash \Omega$ is locally connected at every point, while $\partial \Omega$ is not locally connected at the points in $A=\{(0,0, z): 1<z \leq 2\}$. Moreover the points in $A$ are not accessible from $\Omega$. Note that $\Omega$ is homeomorphic to a ball.

The following example shows that $(\mathrm{f}) \nRightarrow(\mathrm{a})$ in $\mathbf{R}^{3}$.

Example 4.10. Let $K \subset \mathbf{R}^{2}$ be given by

$$
K=\bigcup_{j=1}^{\infty}\left(\left(\left[-2^{-j}, 2^{-j}\right] \times\left\{0,2^{-j}\right\}\right) \cup\left(\left\{-2^{-j}, 2^{-j}\right\} \times\left[0,2^{-j}\right]\right)\right),
$$

and let $\Omega \subset \mathbf{R}^{3}$ be given by

$$
\Omega=(-1,1)^{3} \backslash(K \times[0,1)) .
$$

Then $\partial \Omega$ is locally connected, but no point $(0,0, z) \in \partial \Omega$ is locally accessible from $\Omega$ when $0<z \leq 1$. Moreover, $\Omega$ is not finitely connected at these points. Note that $\Omega$ is homeomorphic to a ball.

By changing the shape of the removed set, we obtain the following example which shows that $(\mathrm{a}) \nRightarrow(\mathrm{d})$ in $\mathbf{R}^{3}$.

Example 4.11. Let $K \subset \mathbf{R}^{2}$ be given by

$$
K=\bigcup_{j=2}^{\infty} \partial B\left(\left(0,2^{-j}\right), 2^{-j}\right)
$$

and let $\Omega \subset \mathbf{R}^{3}$ be given by

$$
\Omega=(-1,1)^{3} \backslash(K \times[0,1)) .
$$

Then any boundary point $x_{0} \in \partial \Omega$ is locally accessible from $\Omega$, but $\Omega$ is not finitely connected at any boundary point $(0,0, z)$ with $0<z \leq 1$. Note that $\Omega$ is homeomorphic to a ball. 
Proof of Theorem 4.2. (e) $\Leftrightarrow(\mathrm{d}) \Rightarrow$ (c) $\Leftrightarrow$ (b) $\Leftrightarrow$ (a) and (f) $\Rightarrow$ (g) These implications follow from Theorem 4.3 (or more directly from Theorems 1.1 and 4.12).

Case 1 . The special case when $\Omega$ is simply connected.

(a) $\Rightarrow(\mathrm{e})$ This is proved in Newman [24, p. 183] (but does not appear in the second edition of [24]).

(a) $\Leftrightarrow$ (f) This is in Newman [24, p. 187, Theorem 12.2] (but not in the second edition of [24]).

$(\mathrm{g}) \Rightarrow$ (f) This is part of Pommerenke [27, p. 20, Theorem 2.1].

Case 2. The general case when $\Omega$ is finitely connected, i.e. $\mathbf{S}^{2} \backslash \Omega$ has finitely many components.

Let $K$ be one of the components, which must be at a positive distance from the other components. Since the conditions (a)-(d), (f) and (g) are all local, any of these conditions holds for $x \in K$ with respect to $\Omega$ if and only if it holds with respect to $\Omega^{\prime}:=\mathbf{S}^{2} \backslash K$. As $\Omega^{\prime}$ is simply connected, and we have already obtained the equivalence for the simply connected case, we see that (a)-(d), (f) and (g) all are equivalent also in the finitely connected case. That (e) is equivalent to the other statements follows from Theorem 1.1.

Proof of Theorem 4.4. Let us first show that (f) and (g) are false. Since $\Omega$ is bounded and infinitely connected, $\mathbf{R}^{2} \backslash \Omega$ consists of infinitely many bounded components (and one unbounded component). Furthermore, we can choose one point from each of these components to obtain a bounded infinite collection of points. This collection must have an accumulation point $x \in \mathbf{R}^{2}$. But then $\mathbf{R}^{2} \backslash \Omega$ is not locally connected at $x$. It also follows that $\partial \Omega$ is not locally connected at $x$.

(a) $\Rightarrow(d)$ This implication is quite complicated to prove and we postpone it to the next section, where it is obtained as Theorem 5.7.

The remaining implications follow from Theorem 4.3.

It is also of interest to consider the corresponding pointwise statements. Contrary to the global statements we need to consider the different types of local connectedness. The statement (e) above does not have a local version, instead we consider the statement $\left(\mathrm{e}^{\prime}\right)$ below.

Theorem 4.12. Assume that $X$ is a proper locally connected metric space and that $\Omega \subset X$ is open. Let $x_{0} \in \partial \Omega$. Consider the following statements:

$\left(\mathrm{a}^{\prime}\right) x_{0}$ is locally accessible from $\Omega$;

(b1') $\Omega \cup\left\{x_{0}\right\}$ is locally pathconnected at $x_{0}$;

(b2') $\Omega \cup\left\{x_{0}\right\}$ is locally connected at $x_{0}$;

(b3') $\Omega \cup\left\{x_{0}\right\}$ is pathconnected im kleinen at $x_{0}$;

(b4') $\Omega \cup\left\{x_{0}\right\}$ is connected im kleinen at $x_{0}$;

$\left(\mathrm{c}^{\prime}\right)$ for all $r>0$ it is true that $x_{0} \notin \overline{H(r)}$;

( $\left.\mathrm{d}^{\prime}\right) \Omega$ is finitely connected at $x_{0}$;

$\left(\mathrm{e}^{\prime}\right) \Omega$ is locally connected at $x_{0}$;

$\left(\mathrm{f1}^{\prime}\right) \partial \Omega$ is locally pathconnected at $x_{0}$;

$\left(\mathrm{f} 2^{\prime}\right) \partial \Omega$ is locally connected at $x_{0}$;

$\left(\mathrm{f} 3^{\prime}\right) \partial \Omega$ is pathconnected im kleinen at $x_{0}$;

$\left(\mathrm{f} 4^{\prime}\right) \partial \Omega$ is connected im kleinen at $x_{0}$;

$\left(\mathrm{g} 1^{\prime}\right) X \backslash \Omega$ is locally pathconnected at $x_{0}$;

$\left(\mathrm{g} 2^{\prime}\right) X \backslash \Omega$ is locally connected at $x_{0}$;

$\left(\mathrm{g} 3^{\prime}\right) X \backslash \Omega$ is pathconnected im kleinen at $x_{0}$;

$\left(\mathrm{g} 4^{\prime}\right) X \backslash \Omega$ is connected im kleinen at $x_{0}$.

Then the following implications are true:

$\left(\mathrm{e}^{\prime}\right) \Longrightarrow\left(\mathrm{d}^{\prime}\right) \Longrightarrow\left(\mathrm{c}^{\prime}\right) \Longleftrightarrow\left(\mathrm{b} 1^{\prime}\right) \Longleftrightarrow\left(\mathrm{b} 2^{\prime}\right) \Longleftrightarrow\left(\mathrm{b} 3^{\prime}\right) \Longleftrightarrow\left(\mathrm{b} 4^{\prime}\right) \Longleftrightarrow\left(\mathrm{a}^{\prime}\right)$ 
and

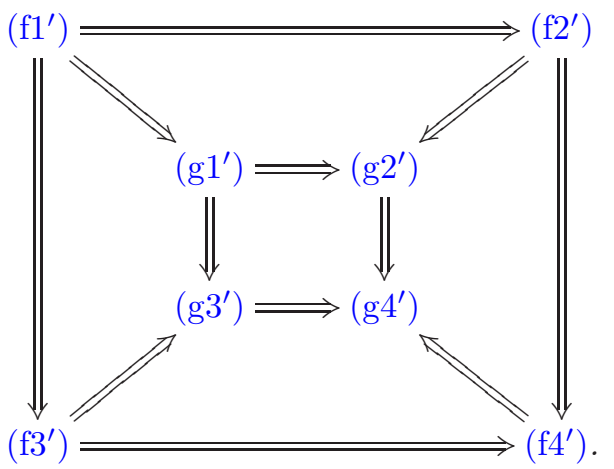

No other implication is true even if we assume that $\Omega$ is a simply connected subset of $\mathbf{R}^{2}$.

Proof of Theorem 4.12. $\left(\mathrm{a}^{\prime}\right) \Rightarrow\left(\mathrm{b}^{\prime}\right)$ If $x_{0}$ is locally accessible from $\Omega$, then for every $r>0$ there exists a positive number $\delta$ such that each $y \in B\left(x_{0}, \delta\right) \cap \Omega$ can be connected by a curve $\gamma_{y}$ in $B\left(x_{0}, r\right) \cap \Omega$ to $x_{0}$. If $y_{1}, y_{2} \in B\left(x_{0}, \delta\right) \cap \Omega$, then the concatenation (at $x_{0}$ ) of the two curves $\gamma_{y_{1}}$ and $\gamma_{y_{2}}$ gives a curve in $B\left(x_{0}, r\right) \cap\left(\Omega \cup\left\{x_{0}\right\}\right)$ connecting $y_{1}$ with $y_{2}$. It follows that the pathcomponent $x_{0}$ belongs to in $B\left(x_{0}, r\right) \cap\left(\Omega \cup\left\{x_{0}\right\}\right)$ contains $B\left(x_{0}, \delta\right) \cap\left(\Omega \cup\left\{x_{0}\right\}\right)$. By the local pathconnectedness of $X$, it now follows that this pathcomponent is a relatively open subset of $\Omega \cup\left\{x_{0}\right\}$, whence we know that $\Omega \cup\left\{x_{0}\right\}$ is locally pathconnected at $x_{0}$. (2.1).

$\left(\mathrm{b} 1^{\prime}\right) \Rightarrow\left(\mathrm{b} 2^{\prime}\right) \Rightarrow\left(\mathrm{b} 4^{\prime}\right)$ and $\left(\mathrm{b} 1^{\prime}\right) \Rightarrow\left(\mathrm{b} 3^{\prime}\right) \Rightarrow\left(\mathrm{b} 4^{\prime}\right)$ These implications follow from

$\left(\mathrm{b} 4^{\prime}\right) \Rightarrow\left(\mathrm{a}^{\prime}\right)$ This follows from Whyburn [30, p. 111, Theorem 4.1] (which should be applied to the component of $X$ containing $x_{0}$ ). Observe that Whyburn's definition of local connectedness is the same as our definition of connectedness im kleinen.

$\neg\left(\mathrm{c}^{\prime}\right) \Rightarrow \neg\left(\mathrm{b} 2^{\prime}\right)$ Let $r>0$ be such that $x_{0} \in \overline{H(r)}$, and let $G \subset B\left(x_{0}, r\right)$ be a neighbourhood of $x_{0}$ in the relative topology of $\Omega \cup\left\{x_{0}\right\}$. Then $G \cap H(r)$ must have infinitely many components, none of which has $x_{0}$ in its boundary. Each of these components is also a component in $G$, which is thus not connected. Hence we have shown that $B\left(x_{0}, r\right) \cap\left(\Omega \cup\left\{x_{0}\right\}\right)$ does not have a (relatively) open connected subset containing $x_{0}$.

$\left(\mathrm{c}^{\prime}\right) \Rightarrow\left(\mathrm{b} 2^{\prime}\right)$ Fix $s>0$. Then by hypothesis $x_{0} \notin \overline{H(s)}$ and hence there is $0<t<s$ such that $B\left(x_{0}, t\right) \cap H(s)=\varnothing$. Let $G=\bigcup_{j=1}^{N(s)} G_{j}(s)$ (where $N(s)$ is either a positive integer or $\infty)$. Then

$$
B\left(x_{0}, t\right) \cap \Omega \subset G \subset B\left(x_{0}, s\right) \cap \Omega .
$$

So $G^{\prime}=G \cup\left\{x_{0}\right\}$ is a neighbourhood of $x_{0}$ in $\Omega \cup\left\{x_{0}\right\}$. We now show that $G^{\prime}$ is connected. Since $x_{0}$ is in the boundary of each $G_{j}(s)$ and $G_{j}(s)$ is connected, it follows that $G_{j}(s) \cup\left\{x_{0}\right\}$ is connected. As $G^{\prime}$ is the union of a collection of connected sets, whose intersection contains $x_{0}$, we see that $G^{\prime}$ is connected as well, see e.g. Lemma 5.1 .

$\left(\mathrm{d}^{\prime}\right) \Rightarrow\left(\mathrm{c}^{\prime}\right)$ This follows from Proposition 2.5.

$\left(\mathrm{e}^{\prime}\right) \Rightarrow\left(\mathrm{d}^{\prime}\right)$ This follows directly from the definition, and we have thus completed the proof of the first set of relations claimed in this theorem.

The implications $\left(\mathrm{f} 1^{\prime}\right) \Rightarrow\left(\mathrm{f} 2^{\prime}\right) \Rightarrow\left(\mathrm{f} 4^{\prime}\right),\left(\mathrm{f} 1^{\prime}\right) \Rightarrow\left(\mathrm{f} 3^{\prime}\right) \Rightarrow\left(\mathrm{f} 4^{\prime}\right),\left(\mathrm{g} 1^{\prime}\right) \Rightarrow\left(\mathrm{g} 2^{\prime}\right) \Rightarrow$ $\left(\mathrm{g} 4^{\prime}\right)$ and $\left(\mathrm{g} 1^{\prime}\right) \Rightarrow\left(\mathrm{g} 3^{\prime}\right) \Rightarrow\left(\mathrm{g} 4^{\prime}\right)$ follow from $(2.1)$.

$\left(\mathrm{f} 1^{\prime}\right) \Rightarrow\left(\mathrm{g} 1^{\prime}\right)$ Let $r>0$. Then there is an open set $G$, with $x_{0} \in G \subset B\left(x_{0}, r\right)$, such that $G \cap \partial \Omega$ is pathconnected. Let $\widetilde{G}$ be the pathcomponent of $G$ containing $G \cap \partial \Omega$. As $X$ is locally connected, $\widetilde{G}$ is open and moreover contains $x_{0}$. Let 
$F=\widetilde{G} \backslash \Omega$. We now show that $F$ is pathconnected. Let $a, b \in F$. Then there is a path $\gamma$ in $\widetilde{G}$ connecting $a$ to $b$. If $\gamma$ does not intersect $\Omega$, then $\gamma$ lies in $F$, and so there is a path in $F$ connecting $a$ to $b$. If $\gamma$ intersects $\Omega$, then it intersects $\partial \Omega$. Let $z$ and $w$ be the first and last time $\gamma$ intersects the closed set $\partial \Omega$. Then $z, w \in \widetilde{G} \cap \partial \Omega=F \cap \partial \Omega$, and so by the pathconnectedness of $G \cap \partial \Omega=\widetilde{G} \cap \partial \Omega$ there is a path $\beta$ connecting $z$ to $w$ in $\widetilde{G} \cap \partial \Omega \subset F$. Let $\gamma_{z}$ and $\gamma_{w}$ be the subcurves of $\gamma$ that lie in $F$ and connect $a$ to $z$ and $b$ to $w$ respectively. The concatenation of $\gamma_{z}, \beta$ and $\gamma_{w}$ is a path in $F$ connecting $a$ to $b$. Hence $F$ is pathconnected and relatively open in $X \backslash \Omega$, and so $X \backslash \Omega$ is locally pathconnected at $x_{0} \in \partial \Omega$.

$\left(\mathrm{f} 2^{\prime}\right) \Rightarrow\left(\mathrm{g} 2^{\prime}\right)$ Let $r>0$. Then there is an open set $G$, with $x_{0} \in G \subset B\left(x_{0}, r\right)$, such that $G \cap \partial \Omega$ is connected. Let $\widetilde{G}$ be the component of $G$ containing $G \cap \partial \Omega$. As $X$ is locally connected, $\widetilde{G}$ is open and moreover contains $x_{0}$. Let $F=\widetilde{G} \backslash \Omega$. We now show that $F$ is connected. Suppose that $F=A_{1} \cup A_{2}$, where $A_{1}$ and $A_{2}$ are disjoint open sets (in the relative topology of $F$ ). Let $F_{1}=A_{1} \cap \partial \Omega$ and $F_{2}=A_{2} \cap \partial \Omega$. Then

$$
F_{1} \cup F_{2}=\widetilde{G} \cap \partial \Omega=G \cap \partial \Omega .
$$

Moreover $F_{1}$ and $F_{2}$ are disjoint and open in the relative topology of $F \cap \partial \Omega=G \cap \partial \Omega$ which is connected. Hence one of them must be empty, say $F_{2}=\varnothing$. It follows that $A_{2}$ and $A_{1}^{\prime}:=A_{1} \cup(\Omega \cap \widetilde{G})$ must be open in the original topology of $X$. As $A_{1}^{\prime}$ and $A_{2}$ are open and disjoint while $\widetilde{G}=A_{1}^{\prime} \cup A_{2}$ is connected, one of them must be empty. Since $x_{0} \in F_{1} \subset A_{1}^{\prime}$, we have $A_{2}$ empty. Thus $F$ is connected and $X \backslash \Omega$ is locally connected at $x_{0} \in \partial \Omega$.

$\left(\mathrm{f} 3^{\prime}\right) \Rightarrow\left(\mathrm{g} 3^{\prime}\right)$ In this case, for each $r>0$ there is a set $E$ and $\delta>0$ such that $B\left(x_{0}, \delta\right) \subset E \subset B\left(x_{0}, r\right)$ and $E \cap \partial \Omega$ is pathconnected. As before, let $\widetilde{E} \subset X$ be the pathcomponent of $E$ containing $x_{0}$ (and hence $E \cap \partial \Omega$ ), and let $F=\widetilde{E} \backslash \Omega$. As in the proof of $\left(\mathrm{f} 1^{\prime}\right) \Rightarrow\left(\mathrm{g} 1^{\prime}\right)$, we see that $F$ is pathconnected. Since $X$ is locally pathconnected, we see that $\widetilde{E}$ contains a neighbourhood of $x_{0}$, and thus $F$ contains a neighbourhood of $x_{0}$ (in the relative topology of $X \backslash \Omega$ ). Hence $X \backslash \Omega$ is pathconnected im kleinen at $x_{0}$.

$\left(\mathrm{f} 4^{\prime}\right) \Rightarrow\left(\mathrm{g} 4^{\prime}\right)$ The proof of this implication is very similar to the proof of $\left(\mathrm{f} 3^{\prime}\right) \Rightarrow$ $\left(\mathrm{g} 3^{\prime}\right)$; just drop the prefix path (from all occurrences) and appeal to (f2') $\Rightarrow\left(\mathrm{g} 2^{\prime}\right)$ instead of $\left(\mathrm{f} 1^{\prime}\right) \Rightarrow\left(\mathrm{g} 1^{\prime}\right)$.

Let us now turn to the counterexamples for the other implications. We present most of them as examples below, but list them here. Note that all counterexamples are with simply connected $\Omega \subset \mathbf{R}^{2}$.

$\left(a^{\prime}\right) \nRightarrow\left(d^{\prime}\right)$ This follows from Example 4.13.

$\left(\mathrm{d}^{\prime}\right) \nRightarrow\left(\mathrm{e}^{\prime}\right)$ This follows from Example 4.14.

$\left(\mathrm{e}^{\prime}\right) \nRightarrow\left(\mathrm{g} 4^{\prime}\right)$ This follows from Example 4.15 with $x_{0}=x_{1}$.

$\left(\mathrm{f} 1^{\prime}\right) \nRightarrow\left(\mathrm{b} 2^{\prime}\right)$ This follows from Example 4.15 with $x_{0}=x_{2}$.

$\left(\mathrm{g} 1^{\prime}\right) \nRightarrow\left(\mathrm{f} 4^{\prime}\right)$ This follows from Example 4.16.

$\left(\mathrm{f} 3^{\prime}\right) \not\left(\mathrm{f} 2^{\prime}\right)$ and $\left(\mathrm{g} 3^{\prime}\right) \not \Rightarrow\left(\mathrm{g} 2^{\prime}\right)$ This follows from Example 4.17.

$\left(\mathrm{f} 2^{\prime}\right) \nRightarrow\left(\mathrm{f} 3^{\prime}\right)$ and $\left(\mathrm{g} 2^{\prime}\right) \not\left(\mathrm{g} 3^{\prime}\right)$ This follows from Example 4.18.

The failure of the remaining implications follows form the above examples in combination with the implications obtained in the beginning of the proof.

Example 4.13. Here we use complex notation. Let $\Omega \subset \mathbf{R}^{2}$ be given by

$$
\Omega:=(0,2)^{2} \backslash \bigcup_{j=1}^{\infty}\left\{r e^{i / j}: 0 \leq r \leq 1\right\}
$$

Then $(0,0)$ is locally accessible from $\Omega$, while $\Omega$ is not finitely connected at 0 . Note that $\Omega$ is simply connected. 
Example 4.14. Let $\Omega$ be the slit disc $B((0,0), 1) \backslash((-1,0] \times\{0\}) \subset \mathbf{R}^{2}$. Then $\Omega$ is finitely connected at the boundary but not locally connected at the boundary. Note that $\Omega$ is simply connected.

Example 4.15. (The topologist's comb II) Let $\Omega \subset \mathbf{R}^{2}$ be given by

$$
\Omega:=(0,2)^{2} \backslash\left(\left\{1, \frac{1}{2}, \frac{1}{3}, \ldots,\right\} \times(0,1]\right),
$$

$x_{1}=(0,1)$ and $x_{2}=(0,0)$. Then $\Omega$ is locally connected at $x_{1}$, while $X \backslash \Omega$ is not connected im kleinen at $x_{1}$. Moreover, $\Omega \cup\left\{x_{2}\right\}$ is not locally connected at $x_{2}$, while $\partial \Omega$ is locally pathconnected at $x_{2}$. Note that $\Omega$ is simply connected.

Example 4.16. Let $\Omega \subset \mathbf{R}^{2}$ be given by

$$
\Omega:=(0,2)^{2} \backslash\left((0,1] \times \bigcup_{j=1}^{\infty}\left[2^{-2 j-1}, 2^{-2 j}\right]\right)
$$

Then $X \backslash \Omega$ is locally pathconnected at $(0,0)$, while $\partial \Omega$ is not connected im kleinen at $(0,0)$. Note that $\Omega$ is simply connected.

Example 4.17. Let $L(a, b)$ be the closed line segment between $a$ and $b$ in $\mathbf{R}^{2}$. Let

$$
E=L((0,0),(1,0)) \cup \bigcup_{j, k=0}^{\infty} L\left(\left(2^{-j-1}, 2^{-j-k}\right),\left(2^{-j}, 0\right)\right) \quad \text { and } \quad \Omega=[-1,1]^{2} \backslash E \text {. }
$$

Then $\partial \Omega$ and $X \backslash \Omega$ are pathconnected im kleinen at $(0,0)$, while neither of them is locally connected at $(0,0)$. Note that $\Omega$ is simply connected and its boundary consists of a chain of "brooms" near the origin. This example can, e.g., be found in Munkres [21, Figure 25.1].

Example 4.18. For $k=1,2, \ldots$, let

$$
\begin{aligned}
E_{k} & =\left\{\left(x, 4^{-k} \sin \left(\pi /\left(4^{k} x-1\right)\right)\right): 4^{-k}<x \leq 2 \cdot 4^{-k}\right\}, \\
F & =\{(0,0)\} \cup \bigcup_{j=1}^{\infty}\left(E_{j} \cup\left(\left[\frac{1}{2} \cdot 4^{-j}, 4^{-j}\right] \times\{0\}\right) \cup\left(\left\{4^{-j}\right\} \times\left[-4^{-j}, 4^{-j}\right]\right)\right), \\
\Omega & =[-1,1]^{2} \backslash F .
\end{aligned}
$$

Then $\partial \Omega$ and $X \backslash \Omega$ are locally connected at $(0,0)$, while neither of them is pathconnected im kleinen at $(0,0)$. Note that $\Omega$ is simply connected.

\section{Countably connected planar domains}

In this section, we shall prove the implication $(\mathrm{a}) \Rightarrow(\mathrm{d})$ (local accessibility of boundary points from $\Omega$ implies finite connectedness of $\Omega$ at the boundary) of Theorem 4.4, viz. Theorem 5.7.

Note that Example 4.11 shows that the implication $(\mathrm{a}) \Rightarrow(\mathrm{d})$ can fail even if $\Omega \subset \mathbf{R}^{3}$ is homeomorphic to a ball. Thus, the arguments in this section cannot be applied to the higher-dimensional case.

The following general topological lemma provides us with a sufficient condition for connectedness of unions of connected sets, and in fact we have already used a special case of it in the proof of Theorem 4.12.

Lemma 5.1. Let $E_{0}$ and $E_{\alpha}, \alpha \in A$, be connected sets. Assume that $E_{0} \cap \bar{E}_{\alpha}$ is nonempty for every $\alpha \in A$. Then $E=E_{0} \cup \bigcup_{\alpha \in A} E_{\alpha}$ is connected. 
This lemma can be found in Moore [20, Theorem I.30, p. 11], but in the spirit of a discussion using modern definitions, we provide a proof of the lemma below.

We alert the reader that the terminology in Moore [20] is somewhat nonstandard. In particular, by compact sets [20] means sequentially precompact sets (which for $\mathbf{R}^{2}$ reduces to bounded sets), and a continuum is a closed connected set (not necessarily compact nor nondegenerate). We will not use such archaic terminology.

Proof. Let $x \in E_{0}$ and let $F$ be the component of $E$ containing $x$. As $E_{0}$ is connected we must have $E_{0} \subset F$. Next, pick an arbitrary $\alpha \in A$. Since $E_{\alpha}$ is connected, its closure relative to $E$ is also connected. This closure contains a point $z \in E_{0}$ and hence must be contained in the component of $z$, which is $F$. In particular $E_{\alpha} \subset F$. Since $\alpha$ was arbitrary we see that $E=F$ and hence $E$ is connected.

In order to obtain our results in this section we deal with upper semicontinuous collections, which we now define.

Definition 5.2. Let $\mathcal{G}$ be a collection of pairwise disjoint compact subsets of $\mathbf{S}^{2}$. Then $\mathcal{G}$ is upper semicontinuous if for every sequence $\left\{K_{j}\right\}_{j=1}^{\infty} \subset \mathcal{G}$ and every set $K \in \mathcal{G}$ it is true that if $x_{j}, y_{j} \in K_{j}, j=1,2, \ldots$, are two sequences such that $x_{j} \rightarrow x$ as $j \rightarrow \infty$ for some $x \in K$, then there exists a subsequence $\left\{y_{j_{k}}\right\}_{k=1}^{\infty}$ of $\left\{y_{j}\right\}_{j=1}^{\infty}$ and some $y \in K$ such that $y_{j_{k}} \rightarrow y$ as $k \rightarrow \infty$.

We will need the following sufficient conditions for upper semicontinuous collections.

Theorem 5.3. The following are true:

(a) The components of a compact subset $F$ of $\mathbf{S}^{2}$ form an upper semicontinuous collection.

(b) A subcollection of an upper semicontinuous collection is upper semicontinuous.

(c) If $\mathcal{G}$ is an upper semicontinuous collection and $K^{\prime}$ is a compact set such that

$$
K^{\prime} \cap \bigcup_{K \in \mathcal{G}} K=\varnothing \text { and } K^{\prime} \cup \bigcup_{K \in \mathcal{G}} K \text { is compact, }
$$

then $\mathcal{G} \cup\left\{K^{\prime}\right\}$ is upper semicontinuous.

Part (a) can be found in Moore [20, Theorem V.1.20, p. 284], but we include a proof here using modern terminology.

Proof. We will first prove (a). To do so we employ the fact that an infinite sequence of compact subsets of $\mathbf{S}^{2}$ has a subsequence that converges in the Hausdorff topology to a compact set, see e.g. Lemma 5.31 in Bridson-Haefliger [7].

Let $\left\{K_{j}\right\}_{j=1}^{\infty} \subset \mathcal{G}$ and $K \in \mathcal{G}$, where $\mathcal{G}$ is the collection of the components of $F$. Next, let $x_{j}, y_{j} \in K_{j}, j=1,2, \ldots$, be two sequences such that $x_{j} \rightarrow x$ as $j \rightarrow \infty$, for some $x \in K$.

By passing to a subsequence if necessary, we may assume that $K_{j} \rightarrow L$ in the Hausdorff topology for some compact set $L$. We now show that $L$ has to be connected. If $L$ is not connected, then there are two open sets $U$ and $V$ such that $L \subset U \cup V, L \cap U$ and $L \cap V$ are nonempty but $L \cap U \cap V$ is empty. Since $L$ is compact and $U$ and $V$ are open, it follows that $L \backslash V=L \cap U=: A$ and $L \backslash U=L \cap V=: B$ are compact and disjoint. Hence there is some $\varepsilon>0$ such that the $\varepsilon$-neighbourhoods of $A$ and $B$ are disjoint. On the other hand, since $K_{j} \rightarrow L$ in the Hausdorff topology, for sufficiently large $j$ we have that $K_{j}$ is contained in the $\varepsilon$-neighbourhood of $L=A \cup B$, which violates the fact that $K_{j}$ is connected. Thus we conclude that $L$ is also connected.

Now we have $x \in L \cap K$, which as a consequence means that $L \cup K$ is also connected, by Lemma 5.1. Since $K$ is a component of $F$ and $L \subset F$ (because 
$F$ is closed), it follows that $L \subset K$. On the other hand, by passing to a further subsequence if necessary and using that $F$ is compact we may assume that $y_{j} \rightarrow y$ for some $y \in F$. Thus $y \in L \subset K$ showing that the collection is upper semicontinous.

The claim (b) is easily seen to be true.

To prove (c), note that the upper semicontinuity condition is satisfied if the "limiting set" $K \in \mathcal{G}$. Now suppose that $K_{j}, j=1,2, \ldots$, are sets from the collection $\mathcal{G}$ and that for each $j$ there is a point $x_{j} \in K_{j}$ such that $x_{j} \rightarrow x_{\infty} \in K^{\prime}$. If $y_{j} \in K_{j}$ as well, then because $K^{\prime} \cup \bigcup_{K \in \mathcal{G}} K$ is compact, it follows that there is a subsequence $\left\{y_{j_{k}}\right\}_{k=1}^{\infty}$ and a point $y_{\infty} \in K^{\prime} \cup \bigcup_{K \in \mathcal{G}} K$ with $y_{j_{k}} \rightarrow y_{\infty}$ as $k \rightarrow \infty$. If $y_{\infty} \in K$ for some $K \in \mathcal{G}$, then by the upper semicontinuity of the collection $\mathcal{G}$ we have that $x_{\infty} \in K$, which violates the assumption that $K^{\prime} \cap K=\varnothing$. Hence $y_{\infty} \in K^{\prime}$. Thus $\mathcal{G} \cup\left\{K^{\prime}\right\}$ is also upper semicontinuous.

The following is our main separation theorem. Recall that a compact set $K \subset \mathbf{S}^{2}$ separates $x$ and $y$ if they belong to different components of $\mathbf{S}^{2} \backslash K$.

Theorem 5.4. Let $T$ be a compact connected subset of $\mathbf{S}^{2}$, and $\mathcal{K}:=\left\{K_{j}\right\}_{j}$ be a nonempty finite or countable upper semicontinuous collection of pairwise disjoint compact sets such that $\bigcup_{j} K_{j}$ is compact.

Let $x, y \in \mathbf{S}^{2} \backslash\left(T \cup \bigcup_{j} K_{j}\right)$. If for each $j$, the set $T \cup K_{j}$ does not separate $x$ from $y$, then $T \cup \bigcup_{j} K_{j}$ does not separate $x$ and $y$ either.

In the special case when $T=\varnothing$ and all the $K_{j}$ are connected this follows from Moore [20, Theorem V.2.3, p. 312].

In order to prove this separation theorem we need to use the following result.

Theorem 5.5. (Janiszewski's theorem) If $F_{1}, F_{2} \subset \mathbf{S}^{2}$ are compact and such that $F_{1} \cap F_{2}$ is connected, and $x, y \in \mathbf{S}^{2} \backslash\left(F_{1} \cup F_{2}\right)$ are not separated by $F_{1}$ nor by $F_{2}$, then they are not separated by $F_{1} \cup F_{2}$.

This result was obtained by Janiszewski [14, Theorem (Twierdwenie) A, p. 48]. For a proof in English see, e.g., Moore [20, Theorem IV.20, p. 173], Newman [24, Corollary to Theorem 8.1, p. 101] or Pommerenke [26, Theorem 1.9].

We also use the following simple lemma.

Lemma 5.6. Assume that $F_{0} \supset F_{1} \supset \ldots$ are compact sets which separate $x$ and $y$. Then so does $F:=\bigcap_{j=0}^{\infty} F_{j}$.

Proof. Suppose not. Then (since connectedness and pathconnectedness coincide for open sets) there is a curve $\gamma$ in the open set $\mathbf{S}^{2} \backslash F$ connecting $x$ and $y$. Because $\left\{F_{j}\right\}_{j=0}^{\infty}$ is a countable decreasing chain of compact sets, and $\gamma$ is also compact and disjoint from $F$, it follows from Cantor's encapsulation theorem (which is sometimes called the finite intersection property) that $\gamma$ lies in the complement of some $F_{k}$, that is, $F_{k}$ does not separate $x$ from $y$. This is a contradiction and hence $F$ separates $x$ and $y$.

Proof of Theorem 5.4. The case when $\mathcal{K}$ is finite follows directly from iteration of Janiszewski's theorem, and we assume therefore that $\mathcal{K}=\left\{K_{j}\right\}_{j=0}^{\infty}$ is infinite in the rest of the proof.

We will prove this theorem by contradiction. So suppose that $x$ and $y$ are separated by $T \cup \bigcup_{j=0}^{\infty} K_{j}$.

Let $\mathcal{C}$ denote the collection of all (necessarily nonempty) compact sets of the form $\bigcup_{j \in A} K_{j}$ such that $x$ and $y$ are separated by $T \cup \bigcup_{j \in A} K_{j}$. We will construct a decreasing sequence in $\mathcal{C}$. Let first $C_{0}=\bigcup_{j=0}^{\infty} K_{j}$ which belongs to $\mathcal{C}$ by assumption. Proceed inductively for $j=0,1, \ldots$ as follows. If $K_{j} \subset C_{j}$ and there is some $C^{\prime} \in \mathcal{C}$ such that $K_{j} \not \subset C^{\prime} \subset C_{j}$, then let $C_{j+1}$ be any such $C^{\prime}$; otherwise, let $C_{j+1}=C_{j}$. 
Let $I=\bigcap_{j=0}^{\infty} C_{j}$. That $I \neq \varnothing$ follows from Cantor's encapsulation theorem (which is sometimes called the finite intersection property). The pairwise disjointness of the sets $K_{j}$ yields that $I$ is of the form $\bigcup_{j \in \tilde{A}} K_{j}$ for some (nonempty) $\tilde{A}$. Let $\widetilde{\mathcal{K}}:=\left\{K_{j}: j \in \tilde{A}\right\}$. Lemma 5.6 applied to $F_{j}=T \cup C_{j}$ shows that $I \in \mathcal{C}$.

Claim. There exists some $K_{m} \in \widetilde{\mathcal{K}}$ for which $\overline{\bigcup_{K \in \widetilde{\mathcal{K}} \backslash\left\{K_{m}\right\}} K}$ and $K_{m}$ are disjoint (that is, $K_{m}$ is isolated from the union of all the other $K \in \widetilde{\mathcal{K}}$ ).

As $T \cup K_{m}$ does not separate $x$ and $y, \widetilde{\mathcal{K}}$ has at least two elements. Since the $K_{j}$ are pairwise disjoint and compact, the claim is trivial if $\tilde{A}$ is finite, and hence we may assume that $\tilde{A}$ is infinite. In order to show the claim we will argue by contradiction and show that then $\widetilde{\mathcal{K}}$ is an infinite compact perfect Hausdorff topological space, when equipped with the topology below. Since such spaces are uncountable (see e.g. Hocking-Young $[13$, p. 88 , Theorem 2-80]), the fact that $\widetilde{\mathcal{K}}$ is countable causes a contradiction, thus showing that the claim is true.

Assume therefore that the claim is false. (In fact we will only use this assumption in Step 3 below.) We equip $\widetilde{\mathcal{K}}$ with a topology as follows. A sequence $\left\{K_{j}^{\prime}\right\}_{j=1}^{\infty}$ in $\widetilde{\mathcal{K}}$ is said to converge to $K^{\prime} \in \widetilde{\mathcal{K}}$ if

$$
\lim _{j \rightarrow \infty} \inf \left\{\varepsilon>0: K_{j}^{\prime} \subset \bigcup_{x \in K^{\prime}} B(x, \varepsilon)\right\}=0 .
$$

To obtain a topology on $\widetilde{\mathcal{K}}$ we say that a set $\mathcal{F} \subset \widetilde{\mathcal{K}}$ is closed if whenever $\left\{K_{j}^{\prime}\right\}_{j=1}^{\infty}$ is a sequence in $\mathcal{F}$ converging to some $K^{\prime} \in \widetilde{\mathcal{K}}$, then $K^{\prime} \in \mathcal{F}$.

Step 1. $\widetilde{\mathcal{K}}$ is Hausdorff. If $K_{1}^{\prime}, K_{2}^{\prime} \in \widetilde{\mathcal{K}}$ are two distinct sets, then choose $0<\varepsilon<\frac{1}{2} \operatorname{dist}\left(K_{1}^{\prime}, K_{2}^{\prime}\right)$ (which is positive since $K_{1}^{\prime}$ and $K_{2}^{\prime}$ are disjoint and compact). Let $\mathcal{O}_{1}$ be the collection of all $K \in \widetilde{\mathcal{K}}$ for which $K \subset \bigcup_{x \in K_{1}^{\prime}} B(x, \varepsilon)=: U$. In order to show that $\mathcal{O}_{1}$ is open, let $\left\{K_{j}^{\prime \prime}\right\}_{j=1}^{\infty}$ be a sequence in $\widetilde{\mathcal{K}} \backslash \mathcal{O}_{1}$ and assume that $K_{j}^{\prime \prime} \rightarrow K^{\prime \prime} \in \widetilde{\mathcal{K}}$. Then there are points $x_{j} \in K_{j}^{\prime \prime} \backslash U$. As $\bigcup_{K \in \widetilde{\mathcal{K}}} K \backslash U=I \backslash U$ is compact, it contains a point $x$ such that a subsequence of $\left\{x_{j}\right\}_{j=1}^{\infty}$ converges to $x$. Since $x_{j} \in K_{j}^{\prime \prime} \rightarrow K^{\prime \prime}$ and $K^{\prime \prime}$ is compact, we conclude from (5.1) that $x \in K^{\prime \prime}$ and therefore $K^{\prime \prime} \notin \mathcal{O}_{1}$, showing that the complement of $\mathcal{O}_{1}$ is closed and thus that $\mathcal{O}_{1}$ is open.

Similarly, let $\mathcal{O}_{2}$ be the collection of all $K \in \widetilde{\mathcal{K}}$ for which $K \subset \bigcup_{x \in K_{2}^{\prime}} B(x, \varepsilon)$, which is also open and disjoint from $\mathcal{O}_{1}$. Moreover $K_{1}^{\prime} \in \mathcal{O}_{1}$ and $K_{2}^{\prime} \in \mathcal{O}_{2}$. We have thus shown that the topology on $\widetilde{\mathcal{K}}$ is Hausdorff.

Step 2. $\widetilde{\mathcal{K}}$ is compact. Let $\left\{\mathcal{G}_{\alpha}\right\}_{\alpha}$ be a (possibly uncountable) cover of $\widetilde{\mathcal{K}}$ by open sets. For each $j \in \tilde{A}$ there is some element $\mathcal{G}_{\alpha_{j}}$ in this cover such that $K_{j} \in \mathcal{G}_{\alpha_{j}}$. Let $\mathcal{G}_{j}^{\prime}=\bigcup_{k \in \tilde{A}, k \leq j} \mathcal{G}_{\alpha_{k}}$.

Assume that $\widetilde{\mathcal{K}} \backslash \mathcal{G}_{j}^{\prime}$ is nonempty for all $j$. Then there is $K_{j}^{\prime} \in \widetilde{\mathcal{K}} \backslash \mathcal{G}_{j}^{\prime}$. Let $x_{j} \in K_{j}^{\prime}$ be arbitrary. By the compactness of $I=\bigcup_{K \in \widetilde{\mathcal{K}}} K$ there exists some $K^{\prime} \in \widetilde{\mathcal{K}}$ and $x \in K^{\prime}$ such that a subsequence $x_{j_{k}} \rightarrow x$ as $k \rightarrow \infty$. Suppose that $\left\{K_{j_{k}}^{\prime}\right\}_{k=1}^{\infty}$ does not converge to $K^{\prime}$ in the above topology, i.e.

$$
\tau:=\limsup _{k \rightarrow \infty} \inf \left\{\varepsilon>0: K_{j_{k}}^{\prime} \subset \bigcup_{x \in K^{\prime}} B(x, \varepsilon)\right\}>0 .
$$

Then for each $n$ we can find $j_{k_{n}}>n$ such that

$$
K_{j_{k_{n}}}^{\prime} \backslash \bigcup_{x \in K^{\prime}} B\left(x, \frac{1}{2} \tau\right) \neq \varnothing
$$


Let $y_{n}$ be a point in this set. Again by the compactness of $I=\bigcup_{K \in \widetilde{\mathcal{K}}} K$, the sequence $\left\{y_{n}\right\}_{n=1}^{\infty}$ has a converging subsequence, but its limit cannot be in $K^{\prime}$, violating the upper semicontinuity of $\widetilde{\mathcal{K}}$. (That $\widetilde{\mathcal{K}}$ is upper semicontinuous follows from Theorem $5.3(\mathrm{~b})$.) Hence $K_{j_{k}}^{\prime}$ converges to $K^{\prime}$. Note that $K^{\prime}=K_{l}$ for some $l \in \tilde{A}$. By construction $K^{\prime} \in \mathcal{G}_{l}^{\prime}$, and so because $\mathcal{G}_{l}^{\prime}$ is open, the tail end of the sequence $K_{j_{k}}^{\prime}$ must lie in $\mathcal{G}_{l}^{\prime}$, violating the choice of $K_{j_{k}}^{\prime}$.

Thus there is some $j$ such that $\widetilde{\mathcal{K}}=\mathcal{G}_{j}^{\prime}$, from which it follows that $\left\{\mathcal{G}_{k}: k \leq\right.$ $j$ and $k \in \tilde{A}\}$ is a finite subcover of $\left\{\mathcal{G}_{\alpha}\right\}_{\alpha}$ covering $\widetilde{\mathcal{K}}$, and hence $\widetilde{\mathcal{K}}$ is compact.

Step 3. $\widetilde{\mathcal{K}}$ is perfect. Let $K^{\prime} \in \widetilde{\mathcal{K}}$. Then by assumption $K^{\prime}$ is not isolated from $\bigcup_{K \in \widetilde{\mathcal{K}} \backslash\left\{K^{\prime}\right\}} K$. Thus there is a sequence $K_{j}^{\prime} \in \widetilde{\mathcal{K}} \backslash\left\{K^{\prime}\right\}$ and points $x_{j} \in K_{j}^{\prime}$ and $x_{0} \in K^{\prime}$ such that $x_{j} \rightarrow x_{0}$ as $j \rightarrow \infty$. It follows as in Step 2 that $K_{j}^{\prime} \rightarrow K^{\prime}$, showing that $\widetilde{\mathcal{K}}$ is perfect.

The above three steps together indicate that $\widetilde{\mathcal{K}}$ must be uncountable, which is not possible. This completes the proof of the claim.

Now let $K_{m} \in \widetilde{\mathcal{K}}$ be as in the claim, and let $\widetilde{\mathcal{K}}^{\prime}=\widetilde{\mathcal{K}} \backslash\left\{K_{m}\right\}$. It follows that $I^{\prime}:=\bigcup_{K \in \widetilde{\mathcal{K}}^{\prime}} K=I \backslash K_{m}$ is a closed subset of the compact set $I=\bigcup_{K \in \widetilde{\mathcal{K}}} K$, and thus must be compact. As $K_{m} \not \subset I^{\prime} \subset C_{m}$ and $K_{m} \subset I$, it follows from the construction of $C_{m+1}$ that $I^{\prime} \notin \mathcal{C}$, i.e. $T \cup I^{\prime}$ does not separate $x$ and $y$. By assumption neither does $T \cup K_{m}$. As $\left(T \cup K_{m}\right) \cap\left(T \cup I^{\prime}\right)=T$ is connected, it follows from Janiszewski's theorem (Theorem 5.5) that $x$ and $y$ are not separated by $T \cup I$.

This is our final contradiction and we have thus completed the proof.

We now formulate and prove the main result of this section.

Theorem 5.7. Let $\Omega \subset \mathbf{R}^{2}$ be bounded and assume that $\mathbf{S}^{2} \backslash \Omega$ consists of (possibly uncountably many) singleton components and at most countably many continuum components. Let $E$ be the union of these continuum components and assume that $\bar{E} \backslash E=F \cup E^{\prime}$, where $F$ is compact and $E^{\prime}$ is at most countable.

If every boundary point $x_{0} \in \partial \Omega$ is locally accessible from $\Omega$, then $\Omega$ is finitely connected at the boundary.

Proof. Fix $x_{0} \in \partial \Omega$ and let $K$ be the component of $\mathbf{S}^{2} \backslash \Omega$ containing $x_{0}$. Then $\Omega^{\prime}=\mathbf{S}^{2} \backslash K$ is simply connected. We shall show that $\Omega^{\prime}$ satisfies condition (a) of Theorem 4.2 , that is, each $x \in \partial \Omega^{\prime}$ is locally accessible from $\Omega^{\prime}$.

Let therefore $x \in \partial \Omega^{\prime} \subset K$ and $r>0$ be arbitrary. As $x$ is locally accessible from $\Omega$, there exists $0<\delta<r / 2$ such that every $y \in \Omega \cap B(x, 2 \delta)$ can be connected to $x$ by a curve in $\Omega \cap B(x, r)$. We want to show that $x$ is locally accessible also from $\Omega^{\prime}$. Let $y \in\left(\Omega^{\prime} \backslash \Omega\right) \cap B(x, \delta)$ and $z$ be a closest point to $y$ on $\partial \Omega$. Clearly, $z$ belongs to the same component of $\mathbf{S}^{2} \backslash \Omega$ as $y$. In particular, $z \notin K$ and $|y-z|<\operatorname{dist}(y, K) \leq|y-x|<\delta$. Since $z \in \partial \Omega$, there exists $y^{\prime} \in \Omega$ such that $\left|y^{\prime}-z\right|<\operatorname{dist}(y, K)-|y-z| \leq \operatorname{dist}(z, K)$. It follows that the line segment from $y$ to $y^{\prime}$ does not hit $K$ and thus lies entirely in $\Omega^{\prime}$. As $\left|x-y^{\prime}\right| \leq|x-y|+\left|y-y^{\prime}\right|<2 \delta$, there exists a curve in $\Omega \cap B(x, r) \subset \Omega^{\prime} \cap B(x, r)$ connecting $y^{\prime}$ and $x$. Combining it with the line segment from $y$ to $y^{\prime}$ provides a curve in $\Omega^{\prime} \cap B(x, r)$ connecting $y$ to $x$. This shows that $x$ is locally accessible from $\Omega^{\prime}$. Since $\Omega^{\prime}$ is simply connected and satisfies condition (a) of Theorem 4.2, it follows from Theorem 4.2 that $\Omega^{\prime}$ is finitely connected at the boundary, and in particular at $x_{0}$. Our aim is to prove that also $\Omega$ is finitely connected at $x_{0}$.

If $K=\left\{x_{0}\right\}$ is a singleton, then let $r_{0}>0$ be arbitrary and $G:=B\left(x_{0}, r_{0}\right)$. If $K$ is a continuum, then let $r_{0}>0$ be so small that $K \backslash B\left(x_{0}, r_{0}\right) \neq \varnothing$ and let $B_{0}=B\left(x_{0}, r_{0}\right)$. Then there are only finitely many components of $\Omega^{\prime} \cap B_{0}$ with $x_{0}$ in their closure, as $\Omega^{\prime}$ is finitely connected at $x_{0}$. Let $G$ be one of these components. 
We will now show that $G$ is simply connected. Suppose not. Then $\mathbf{S}^{2} \backslash G$ has at least two components, each of which is compact. Let $\widetilde{F}$ be the component of $\mathbf{S}^{2} \backslash G$ containing the connected set $K \cup\left(\mathbf{S}^{2} \backslash B_{0}\right.$ ) (which is connected by Lemma 5.1 and the choice of $r_{0}$ ). Let $\widehat{F}$ be any other component of $\mathbf{S}^{2} \backslash G$. Then clearly $\widehat{F} \subset \Omega^{\prime} \cap B_{0}$. On the other hand, $\widehat{F} \cap \bar{G}$ is nonempty. Therefore by Lemma 5.1 again, $\widehat{F} \cup G$ is a connected subset of $\Omega^{\prime} \cap B_{0}$, which violates the fact that $G$ is a component of $\Omega^{\prime} \cap B_{0}$. Hence $G$ is simply connected. (If $K$ is a singleton, then $G=B_{0}$ is clearly simply connected.)

We shall now prove that $\Omega$ is finitely connected at $x_{0}$. Since $x_{0}$ is locally accessible from $\Omega$, Theorem 4.3 shows that $x_{0} \notin \overline{H_{\Omega}\left(r_{0}, x_{0}\right)}$, i.e. there exists $\delta>0$ such that $B\left(x_{0}, \delta\right) \cap H_{\Omega}\left(r_{0}, x_{0}\right)=\varnothing$. Let $z, w \in G \cap \Omega \cap B\left(x_{0}, \delta\right)$. We will show that $z$ and $w$ cannot be separated in $G \cap \Omega$.

Let $F_{j}, j=1,2 \ldots$, denote all the (at most countably many) continuum components of $\mathbf{S}^{2} \backslash \Omega$ (including $K$ if it is nonsingleton), together with all the (at most countably many) singleton components of $E^{\prime} \backslash F$. Note that $F \subset\left(\mathbf{S}^{2} \backslash \Omega\right) \backslash E$ consists only of singleton components of $\mathbf{S}^{2} \backslash \Omega$ and is therefore totally disconnected. Note also that if $K=\left\{x_{0}\right\}$ is singleton, we may have $x_{0} \in F \cup E^{\prime}$.

Let $T_{j}=\left(F_{j} \cap G\right) \cup\left(\mathbf{S}^{2} \backslash B_{0}\right), j=1,2, \ldots$. We shall show that $T_{j}$ does not separate $z$ and $w$. This is clear if $F_{j}=K$. If $F_{j} \neq K$, then $\operatorname{dist}\left(x_{0}, F_{j}\right)>0$ and hence also $\operatorname{dist}\left(x_{0}, T_{j}\right)>0$. As $z, w \notin H_{\Omega}\left(r_{0}, x_{0}\right)$, they belong to some components of $\Omega \cap B_{0}$ with $x_{0}$ in their closures. Since $\Omega \cap B_{0} \subset B_{0} \backslash F_{j} \subset \mathbf{S}^{2} \backslash T_{j}$, we see that those components of $\Omega \cap B_{0}$ are subsets of $z$ 's and $w$ 's component(s) in $\mathbf{S}^{2} \backslash T_{j}$, which thus must contain $x_{0}$ ( $\operatorname{since} \operatorname{dist}\left(x_{0}, T_{j}\right)>0$ ). But there can only be one such component in $\mathbf{S}^{2} \backslash T_{j}$, so $T_{j}$ does not separate $z$ and $w$.

Clearly, $T:=\mathbf{S}^{2} \backslash G$ does not separate $z$ and $w$. Since $F$ is totally disconnected it has topological dimension zero, see e.g. Fedorchuk [8, Theorem 5 in Section 1.3.2], and thus cannot separate the open set $G$, which has topological dimension 2 , see e.g. [8, Theorem 20 in Section 2.7]. Thus $F \cup T$ does not separate $z$ and $w$. Further, $T_{j}=\left(F_{j} \cap \bar{G}\right) \cup\left(\mathbf{S}^{2} \backslash B_{0}\right)$ is compact and $T_{j} \cap T=\mathbf{S}^{2} \backslash B_{0}$ is connected, so Janiszewski's theorem (Theorem 5.5) implies that $T_{j} \cup T=F_{j} \cup T$ does not separate $z$ and $w$ either.

As $\mathbf{S}^{2} \backslash \Omega$ and each of its components is compact, the collection $\left\{F_{j}\right\}_{j}$ is upper semicontinuous, by Theorem $5.3(\mathrm{a})$ and (b). Theorem 5.3 (c) then yields that $\left\{F_{j}\right\}_{j} \cup\{F\}$ is also an upper semicontinuous collection of pairwise disjoint compact sets. By Theorem 5.4 we now obtain that the set

$$
T \cup \bar{E}=T \cup \bigcup_{j} F_{j} \cup F
$$

does not separate $z$ and $w$. Finally, $\left(\mathbf{S}^{2} \backslash \Omega\right) \backslash \bar{E}$ is totally disconnected, so it does not separate $\mathbf{S}^{2} \backslash(T \cup \bar{E})$, i.e. $z$ and $w$ belong to the same component of

$$
\mathbf{S}^{2} \backslash\left((T \cup \bar{E}) \cup\left(\left(\mathbf{S}^{2} \backslash \Omega\right) \backslash \bar{E}\right)\right)=\mathbf{S}^{2} \backslash\left(T \cup\left(\mathbf{S}^{2} \backslash \Omega\right)\right)=G \cap \Omega \subset \Omega \cap B_{0}
$$

This implies that each component $G$ of $\Omega^{\prime} \cap B_{0}$ with $x_{0}$ in its closure contains exactly one component of $\Omega \cap B_{0}$ with $x_{0}$ in its closure. Indeed, if there were two such components $G_{1}$ and $G_{2}$ of $\Omega \cap B_{0}$, both contained in $G$, then we could find $z \in G_{1} \cap B\left(x_{0}, \delta\right)$ and $w \in G_{2} \cap B\left(x_{0}, \delta\right)$, and the above argument would give that $z$ and $w$ belong to the same component of $\Omega \cap G$, which is a contradiction.

Since $\Omega^{\prime}$ is finitely connected at $x_{0}$ (or $G=B_{0}$ for singleton $K=\left\{x_{0}\right\}$ ), this implies that $\Omega$ is also finitely connected. As $x_{0}$ was arbitrary, this completes the proof. 


\section{References}

1. Adamowicz, T., Buörn, A., Buörn, J. and Shanmugalingam, N., Prime ends for domains in metric spaces, Adv. Math. 238 (2013), 459-505.

2. Aikawa, H. and Hirata, K., Doubling conditions for harmonic measure in John domains, Ann. Inst. Fourier (Grenoble) 58 (2008), 429-445.

3. BJörn, A. and BJöRn, J., Nonlinear Potential Theory on Metric Spaces, EMS Tracts in Mathematics 17, European Math. Soc., Zurich, 2011.

4. Björn, A., Buörn, J. and Shanmugalingam, N., The Dirichlet problem for $p$-harmonic functions on metric spaces, J. Reine Angew. Math. 556 (2003), 173-203.

5. Björn, A., Buörn, J. and Shanmugalingam, N., The Perron method for p-harmonic functions, J. Differential Equations 195 (2003), 398-429.

6. Buörn, A., BuÖRn, J. and Shanmugalingam, N., The Dirichlet problem for $p$-harmonic functions with respect to the Mazurkiewicz boundary, Preprint, 2013, arXiv:1302.3887.

7. Bridson, M. and Haefliger, A., Metric Spaces of Non-positive Curvature, Grundlehren der Mathematischen Wissenschaften 319, Springer, Berlin, 1999.

8. Fedorchuk, V. V., The fundamentals of dimension theory, in General Topology I (Arkhangel'skiŭ, A. V. and Pontryagin, L. S., eds.), Encyclopedia of Mathematical Sciences 17, pp. 111-224, Akad. Nauk SSSR, Vsesoyuz. Inst. Nauchn. i Tekhn. Inform., Moscow, 1988 (Russian). English transl.: pp. 91-192, SpringerVerlag, Berlin-Heidelberg, 1990.

9. Freeman, D. M. and Herron, D. A., Bilipschitz homogeneity and inner diameter distance, J. Anal. Math. 111 (2010), 1-46.

10. Granlund, S., Lindqvist, P. and Martio, O., Note on the PWB-method in the nonlinear case, Pacific J. Math. 125 (1986), 381-395.

11. Heinonen, J., Kilpeläinen, T. and Martio, O., Nonlinear Potential Theory of Degenerate Elliptic Equations, 2nd ed., Dover, Mineola, NY, 2006.

12. Herron, D. A. and Sullivan, T. S., Fractal inner chordarc disks, J. Anal. Math. 84 (2001), 173-205.

13. Hocking, J. G. and Young, G. S., Topology, Dover, New York, 1988.

14. JANiszewski, Z., 0 rozcinaniu płaszczyzny przez kontinua [On separating the plane by continua], Prace Mat.-Fiz. 26 (1915), 11-63 (Polish).

http://matwbn.icm.edu.pl/index.php?jez=en

15. Karmazin, A. P., Quasiisometries, the Theory of Prime Ends and Metric Structures on Domains, Surgut, 2008 (Russian).

16. KilpeläInen, T., Potential theory for supersolutions of degenerate elliptic equations, Indiana Univ. Math. J. 38 (1989), 253-275.

17. Kuratowski, K., Topology, vol. 2, Academic Press, New York-London, 1968.

18. Mazurkiewicz, S., Sur une classification de points situés un sur continu arbitraire [O pewnej klasyfikacyi punktów leżących na kontynuach dowolnych], $C$. R. Soc. Sci. Lett. Varsovie 9:5 (1916), 428-442 (Polish with French summary at the end).

19. Moore, R. L., A connected and regular point set which contains no arc, Bull. Amer. Math. Soc. 32 (1926), 331-332.

20. Moore, R. L., Foundations of Point Set Theory, 2nd ed., Amer. Math. Soc., 1962.

21. Munkres, J. R., Topology, 2nd ed., Prentice-Hall, Upper Saddle River, NJ, 2000.

22. NÄKKI, R., Boundary behavior of quasiconformal mappings in $n$-space, Ann. Acad. Sci. Fenn. Ser. A I Math. 484 (1970), 1-50.

23. NÄkki, R., Private communication, 2010. 
24. Newman, M. H. A., Elements of the Topology of Plane Sets of Points, 1st ed., Cambridge Univ. Press, Cambridge, 1939.

25. Ohtsuka, M., Dirichlet Problem, Extremal Length and Prime Ends, Van Nostrand, Princeton, NJ, 1970.

26. Pommerenke, C., Univalent Functions, Vandenhoeck \& Ruprecht, Göttingen, 1975.

27. Pommerenke, C., Boundary Behaviour of Conformal Maps, Springer, BerlinHeidelberg, 1992.

28. Rempe, L., On prime ends and local connectivity, Bull. Lond. Math. Soc. 40 (2008), 817-826.

29. V̈̈IsÄLÄ, J., Lectures on n-dimensional Quasiconformal Mappings, Lecture Notes in Math. 229, Springer, Berlin-Heidelberg, 1971.

30. Whyburn, G. T., Analytic Topology, Amer. Math. Soc. Colloq. Publ. 28, Amer. Math. Soc., Providence, RI, 1942. 